\title{
PISTIL, a reactivity modulation device to probe the transfer function of the nuclear reactor CROCUS
}

\author{
Yifeng Jiang ${ }^{1,2}$, Benoit Geslot ${ }^{1}$, Vincent Lamirand ${ }^{2}$, Pierre Leconte ${ }^{3}$, Daniel Godat ${ }^{2}$, Laurent Braun ${ }^{2}$, Pavel Frajtag ${ }^{2}$, \\ Christine Coquelet-Pascal ${ }^{1}$ and Andreas Pautz ${ }^{2}$ \\ 1 CEA, DES, IRESNE, DER, SPESI, LP2E, Cadarache, F-13108 Saint-Paul-Lez-Durance, France \\ 2 Laboratory of Reactor Physics and Systems Behaviour, Ecole Polytechique Fédérale de Lausanne, CH-1015 \\ Lausanne, Switzerland \\ 3 CEA, DES, IRESNE, DER, SPRC, LEPh, Cadarache, F-13108 Saint-Paul-Lez-Durance, France \\ Corresponding author: yifeng.jiang@ cea.fr
}

\begin{abstract}
The present article summarizes the development and testing of a reactivity modulation device developed by the French Atomic and Alternative Energies Commission (CEA). It was installed in the CROCUS reactor of the Swiss Federal Institute of Technology in Lausanne (EPFL). Experimental tests were performed in the framework of a collaboration between CEA and EPFL.

The so-called PISTIL device aims at measuring the nuclear reactor transfer function in the frequency range of interest between $1 \mathrm{mHz}$ and $200 \mathrm{~Hz}$, in order to probe the in-core kinetic behavior of prompt and delayed neutrons. The reactivity modulation is obtained from the rotation of cadmium foils.

The design of the system was driven with the objective of installing PISTIL at the center of the CROCUS reactor. Neutronic simulations with TRIPOLI-4 Monte Carlo code were performed to select the suitable design parameters and meet the safety requirements of the reactor operation.

The total reactivity worth of the device, as estimated by TRIPOLI-4 Monte Carlo calculation, was approximately $0.16 \$$ and the maximum amplitude of the reactivity modulation was about $0.013 \$$. In-core reactivity calibration was then performed and were consistent as compared to TRIPOLI-4 estimations.
\end{abstract}

Keywords - In-core integral experiment, reactivity modulation, zero-power reactor transfer function, reactor kinetics

\section{INTRODUCTION}

$\mathrm{T}$ he pile oscillator method is well-known as an experimental approach to evaluate the model consistency of transient calculations in nuclear reactors with respect to its temporal behavior in reactor physics [1], as the corresponding knowledge are crucial to the design, operation and safety analysis of nuclear reactors. Instead of studying the temporal response of the in-core neutron population subject to variations in reactor configuration (e.g. control rod movement, temperature feedback effect, etc.), a periodic reactivity modulation is introduced to investigate the overall reactor kinetic behavior in the frequency domain through the measurement of the reactor transfer function (RTF) by Fourier analysis.

A particular feature of the RTF of low power reactors, namely the Zero-Power reactor Transfer Function (ZPTF), is that it is only dependent on the kinetic behavior of prompt and delayed neutron population produced in the fission chain reaction. While an extensive amount of computational work were conducted to improve the modeling of reactor kinetics, recent studies [2-4] have shown the potential of an alternative experimental approach, through in-core reactivity modulation, towards the better understanding of the underlying physical phenomena. In this context, the PISTIL device (Periodic reactivity Injection System Transients Induced Locally) was developed by CEA in the framework of a collaboration between CEA and EPFL. The device has two aluminum tubes holding cadmium foils. The rotation of the center tube, driven by a brushless motor, generates a controlled reactivity modulation through the variation of the radiative capture reaction rate of the cadmium. The height of the ensemble, as well as the relative position between rotary and static parts are adjustable, offering flexibility on reactivity worth and reactivity amplitude. The reactivity calibration test of the device was performed in the zeropower reactor CROCUS at EPFL for the experimental investigation of its reactor kinetic parameters by frequency domain analysis. In this work, we present the design methodology and first calibration results of this experimental device.

\section{CONTEXT AND BACKGROUND}

\section{A. Point kinetic reactor model and Zero-power transfer function}

The point kinetic model in reactor physics describes the temporal behavior (integrated in space and energy) of the neutron population in the core of a nuclear reactor, with respect to a reactivity variation $[1]$ :

$$
\left\{\begin{array}{l}
\frac{d N(t)}{d t}=\frac{\rho(t)-\sum_{i}^{n} \beta_{i}}{\Lambda} N(t)+\sum_{i}^{n} \lambda_{i} C_{i}(t) \\
\frac{d C_{i}(t)}{d t}=\frac{\beta_{i}}{\Lambda} N(t)-\lambda_{i} C_{i}(t) \quad i=1,2, \ldots, n
\end{array}\right.
$$

Where $N(t)$ represents the neutron population, $\rho(t)$ is the reactivity, $C_{i}(t)$ is the delayed neutron precursor concentration of group $i$ in an n-group formulation. The kinetic parameters involved in this formulation are the prompt neutron generation 
time $\Lambda(\mathrm{s})$, the effective delayed neutron fractions $\left(\beta_{i}\right)_{i=1,2, \ldots, n}$ and associated decay constants $\left(\lambda_{i}\right)_{i=1,2, \ldots, n}\left(\mathrm{~s}^{-1}\right)$ for the precursor group $i$. As mentioned earlier, the temperature feedback effects are considered negligible in the case of zeropower reactors.

When a small and periodic reactivity perturbation $\rho(t)=$ $\rho_{0}+\delta \rho(t)$ is generated in the reactor core, its kinetic behavior with respect to the neutron population $N(t)=N_{0}+\delta N(t)$ can be modelled by a linear time invariant system represented in the frequency domain by the ZPTF [6]:

$$
\frac{N\left(j \omega_{0}\right)}{\rho\left(j \omega_{0}\right) N_{0}}=H\left(j \omega_{0}\right)=\left(j \Lambda \omega_{0}+\sum_{i}^{n} \frac{j \omega_{0} \beta_{i}}{j \omega_{0}+\lambda_{i}}-\rho_{0}\right)^{-1}
$$

Where $j$ is the unit imaginary number and $\omega_{0}$ is the angular frequency of the perturbation $\left(\mathrm{rad} . \mathrm{s}^{-1}\right)$. The function, as in the complex domain, is conventionally represented by its modulus (amplitude) and argument (phase).

The measurement of the ZPTF can be achieved by modulating periodically the reactivity at several given frequencies and measure its effect on the neutron flux. Thanks to standard Fourier analysis techniques, the kinetic parameters are inferred in the frequency domain: the reactivity variation profile and the neutron detector response. An example of simulated modulation experiment and resulting analysis for ZPTF, in a point reactor kinetic formulation manner, is shown in Figure 1. When a sine-like reactivity modulation is introduced to the reactor, a power (neutron flux) variation of similar profile can be measured. The analysis of the amplitude ratio and phase lag is measured at the characteristic fundamental frequency of the modulation.

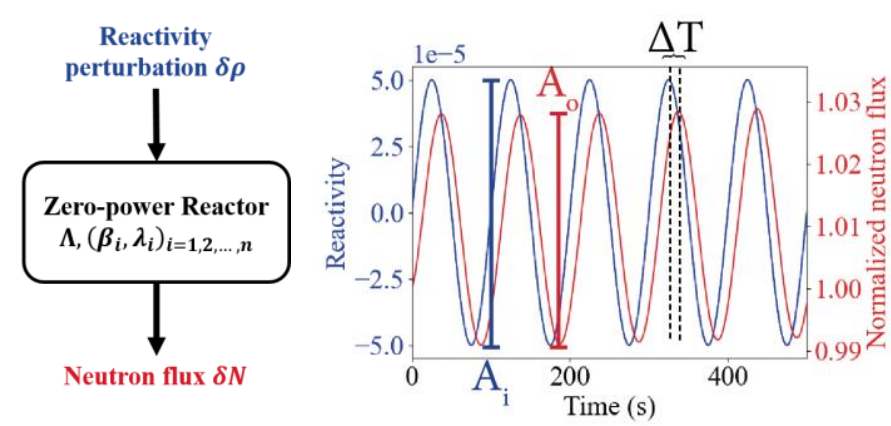

(a) Reactivity perturbation and simulated reactor power response
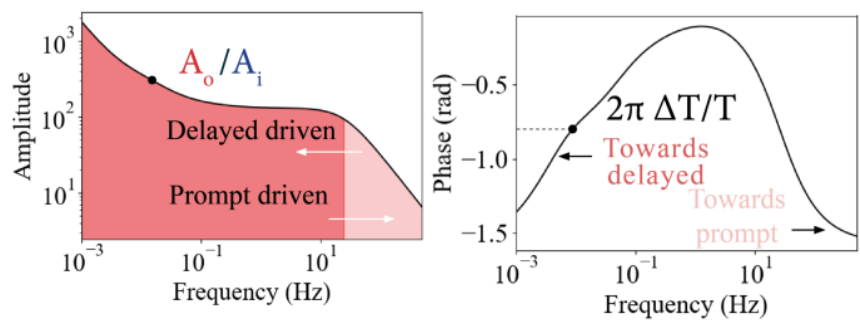

(b) Amplitude and phase representation of the ZPTF in the frequency domain

Figure 1. Simulated zero-power reactor behavior in a modulation experiment

\section{B. The CROCUS reactor}

CROCUS is a zero-power research reactor operated at EPFL.
Figure 2 shows a cross sectional view of the reactor core and its associated structures. The maximum power of the reactor is limited to $100 \mathrm{~W}$, corresponding to a neutron flux of $2.5 \times 10^{9}$ $\mathrm{cm}^{-2} \cdot \mathrm{s}^{-1}$ in the core center. The reactor is operated at atmospheric pressure and room temperature using a controlled water loop. The power is adjusted either by changing the incore water level using a spillway, or by moving two $\mathrm{B}_{4} \mathrm{C}$ control rods.

The reactor core is light-water moderated and uranium fueled. The active zone of the core is approximately a cylinder of $60 \mathrm{~cm}$ in diameter and $1 \mathrm{~m}$ in height. It is composed of two interlocked fuel zones: an inner zone of $336 \mathrm{UO}_{2}$ cylindrical rods enriched to $1.806 \%$ and an outer zone of $176 \mathrm{U}_{\text {metal }}$ cylindrical rods enriched to $0.947 \%$. Two aluminum grid plates spaced $1 \mathrm{~m}$ apart maintain the fuel rods vertically. PISTIL was designed to be held in a similar manner in an experimental channel available at the core-center of $10 \mathrm{~mm}$ diameter. In the following parts of the article, all mentioned axial positions are those of the reference height documented in the CROCUS benchmark [9].

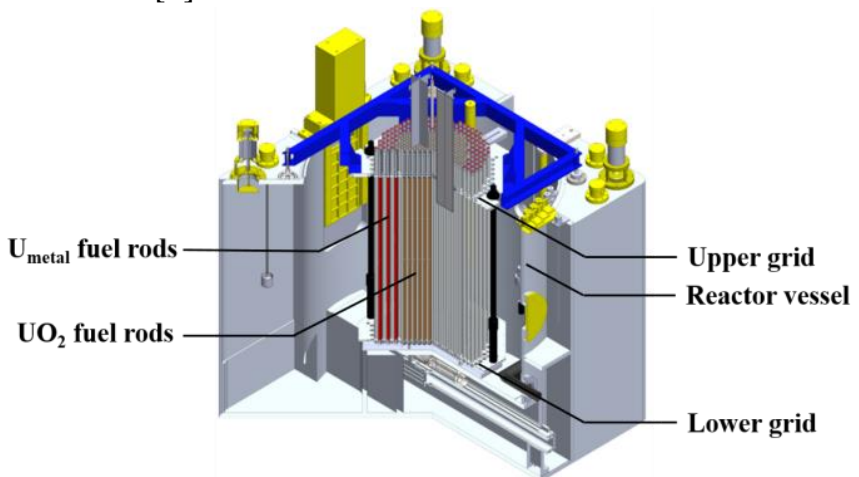

Figure 2. Cross-sectional view of the CROCUS reactor

\section{NEUTRONIC DESIGN}

\section{A. Estimation of the differential reactivity worth}

The neutronic design of the PISTIL device was conducted using the transport Monte Carlo code Tripoli-4® [7]. A twostep approach was adopted for the study.

In the first step, criticality calculations using a reduced scale model, representative of the fuel lattice of the core center, as shown in Figure 3, were first performed with the objective of selecting the best isotopes to achieve the reactivity modulation. A qualitative concept of absorbers in rotation was produced. The reactivity effect are induced by the variation of the distance between two cadmium foils: when they are far from each other, the macroscopic cross sections is maximum. In contrast when they are close, the cross sections decreases due to self-shielding effects.

In the second step, calculations of the whole core of CROCUS, with PISTIL modeled at the core center, were conducted for the estimation of the overall reactivity worth and reactivity modulation amplitude.

Between two different reactor configurations, the differential reactivity worth was estimated as:

$$
\Delta \rho_{1 \rightarrow 2}=\frac{1}{k_{e f f, 1}}-\frac{1}{k_{e f f, 2}}
$$




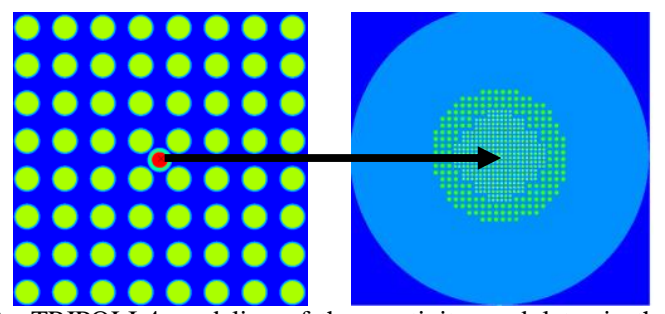

Figure 3. TRIPOLI-4 modeling of the reactivity modulator in the center of reduced scale fuel lattice (left) and in the full core of CROCUS

\section{B. Conceptual design}

The design consists in several cylindrical elements positioned vertically in the reactor core. The main components of the neutronic design of PISTIL, in a cross-sectional view, are shown in Figure 4. The system is composed of a watertight aluminum tube, a static subset (stator) of two cadmium foils and a rotary subset (rotor) composed of another two cadmium foils and a high-density polyethylene (HDPE) cylinder. The change in the relative angular position between the rotor and the stator generates variation in the $(n, \gamma)$ absorption reaction rate [8]. The HDPE has the effect of enhancing the capture reaction rates by inducing a local thermalization of the neutron spectrum. Cadmium was selected as the absorber material for its high $(n, \gamma)$ cross section in the thermal energy range $(<0.5 \mathrm{eV})$ compared to other conventional materials that are easy to machining to foils of less than $0.1 \mathrm{~mm}$ thickness. The foil geometry was chosen to minimize the strong self-shielding effect within the volume of the cadmium material, which reduces considerably the modulation effect.

A rotary motion drives the reactivity modulation induced by the cadmium foil on the rotor. As the two foils on the stator and the rotor cover each one a $90^{\circ}$ section and separated $90^{\circ}$, the device has a 2 -fold rotational symmetry. Such a design allows the doubling of the modulation frequency as compared to the mechanical frequency in concern of relieving safety considerations at frequencies as high as $100 \mathrm{~Hz}$. The rotary motion was preferred than the linear one in this aspect, as it would introduce much less vibration at high frequency.

In the following sections, the $0^{\circ}$ will refer to the angular position configuration of the rotor and stator shown in Figure 4. This corresponds to the minimum reactivity worth of PISTIL. In contrast, the maximum reactivity corresponds to the configuration of a $90^{\circ}$ rotation from the $0^{\circ}$ position.

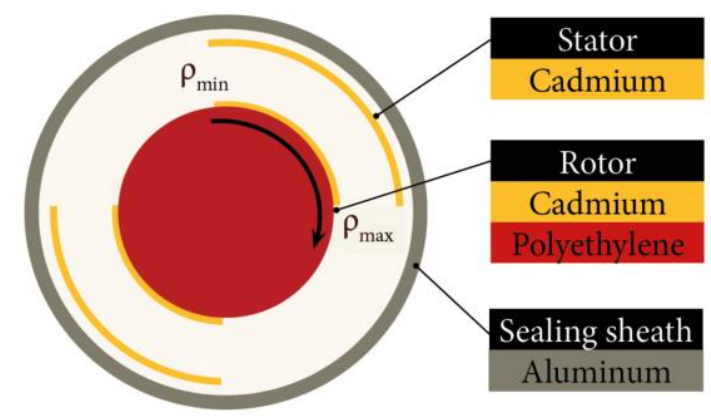

Figure 4. Main components of the neutronic design of PISTIL

\section{Reactivity worth and modulation amplitude}

Full core calculations were performed for several configurations of the reactor. All these calculations use the JEFF 3.3 library [11] for nuclear data, and the in-core water level was considered at $96.5 \mathrm{~cm}$.

The cadmium elements and the HDPE were modelled at the same axial position centered at $550 \mathrm{~cm}$. This configuration will also be considered for the experimental calibration discussed in section $\mathrm{V}$. The calculation-based estimation showed that the expected maximum anti-reactivity insertion is about $0.16 \$$ while the modulation amplitude is $1.3 \varnothing$. The amplitude of the modulation is sufficiently large for the ZPTF measurement in the frequency range of interest.

The choice of the maximum reactivity worth was driven by the limit of the additional reactivity reserve of $0.2 \$$ available in the current configuration of CROCUS [14] to bring the reactor to critical, while optimizing the precision of the measurements by maximizing the modulation amplitude.

TABLE 1

CALCULATED MULTIPLICATION FACTOR IN VARIOUS REACTOR CONFIGURATIONS

\begin{tabular}{cccc}
\hline \hline Configuration & $\mathrm{k}_{\mathrm{eff}}$ & $\begin{array}{c}\text { Relative } \\
\text { uncertainty } \\
(\%)\end{array}$ & $\begin{array}{c}\text { Differential } \\
\text { worth }(\mathrm{pcm})\end{array}$ \\
\hline $\begin{array}{c}\text { Reference without } \\
\text { PISTIL }\end{array}$ & 1.002858 & $2.88189010^{-3}$ & - \\
PISTIL 0 & 1.001729 & $2.4889510^{-3}$ & $-112.4 \pm 3.8$ \\
PISTIL 45 & 1.001688 & $2.4891010^{-3}$ & $-116.4 \pm 3.8$ \\
PISTIL 90 & 1.001628 & $2.4997610^{-3}$ & $-122.4 \pm 3.8$ \\
\hline \hline
\end{tabular}

\section{MechaniCAL DESIGN}

The available in-core space in the center of CROCUS, with a outer diameter of $10 \mathrm{~mm}$ due to the presence of the two grids, led to the design of only including the elements mentioned in the neutronic design in the active core. The other structures of PISTIL and the motor are placed above the fuel zones. Other positioning options of the device less restrictive with respect to the geometry, such as the periphery regions, were discarded due to the insufficient reactivity modulation amplitude.

\section{A. Mechanical components}

The dimensions of the elements present in the fuel zone are shown in Table II. Figure 5 shows a mechanical representation of the whole device.

TABLE II

\begin{tabular}{cccc}
\multicolumn{4}{c}{ DIMENSIONS OF ELEMENTS IN THE NEUTRONIC DESIGN OF PISTIL } \\
\hline \hline Element & $\begin{array}{c}\text { Axial } \\
\text { length } \\
(\mathrm{mm})\end{array}$ & $\begin{array}{c}\text { Outer } \\
\text { diameter } \\
(\mathrm{mm})\end{array}$ & $\begin{array}{c}\text { Inner } \\
\text { diameter } \\
(\mathrm{mm})\end{array}$ \\
\hline Sealing sheath & 1000.0 & 10.0 & 9.0 \\
Cadmium on stator & 100.0 & 8.63 & 8.4 \\
Cadmium on rotor & 100.0 & 5.70 & 5.46 \\
HDPE & 100.0 & 5.50 & 0 \\
\hline \hline
\end{tabular}

The bottom of the device was designed to be in contact with the base plate of CROCUS, while its upper edges are fixed to the reactor's structural support above the core. 


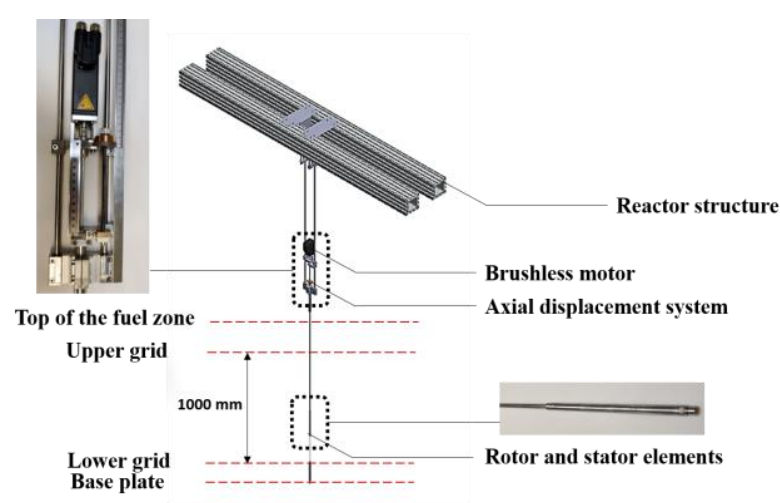

Figure 5. Schematic representation of the mechanical elements of PISTIL

The mechanical concept of PISTIL included the possibility to modify the axial position of the rotor with respect to the stator over $100 \mathrm{~mm}$ range, while the rotor/stator ensemble can also be adjusted from $550 \mathrm{~mm}$ to $950 \mathrm{~mm}$. This can be achieved when the device is installed inside the core.
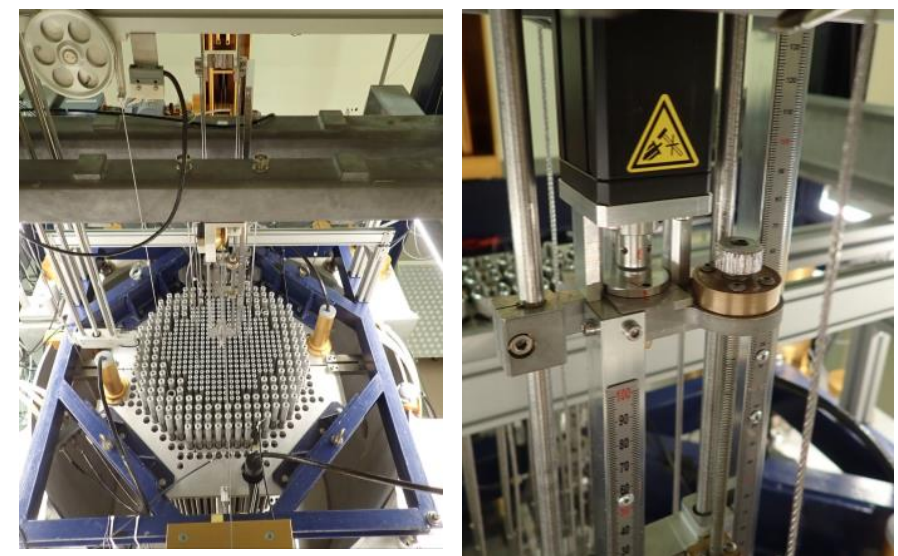

Figure 6. PISTIL installed in the core center of CROCUS (left), its motor and position adjustment system (right)

\section{B. Motion command and data communication system}

A TGS1 brushless motor with a nominal speed of $6000 \mathrm{rpm}$ $(100 \mathrm{~Hz})$ drives the mechanical motion of the rotor of PISTIL. The motion profile is controlled by a $230 \mathrm{~V}$ AC INFRANOR XtrapulsePac servo-controller. The motion commands are communicated through the numerical control TrioMotion Flex6-Nano P600, using a program developed with National Instrument LabView [12] via the ActiveX TRIO PC Motion [10].

A number of motion parameters, such as the angular position and the servo current of the motor are acquired in parallel to the rotation by the numerical control at a maximum sampling rate of $1 \mathrm{kHz}$. These data are received by buffer in the aforementioned LabView program through a User Datagram Protocol (UDP) transmission.

Two types of instructions are currently implemented for the motion of PISTIL. The motor can rotate either continuously at a given frequency, or following a stepwise predefined profile in angular position. In Figure 7, the two modes are illustrated: a continuous rotation (left) and a pseudo-random sequence of quarter flip motions (right). The angular positions are shown in degree $\left(^{\circ}\right)$, while the neutron flux are normalized to its mean value during the experiment.

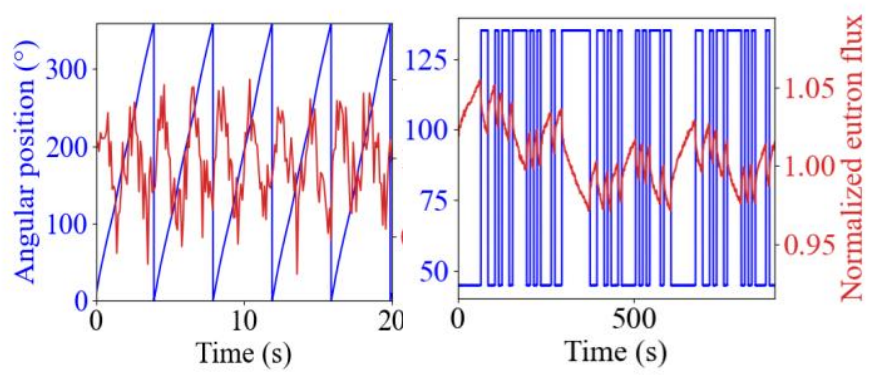

Figure 7. Measured motion profile of PISTIL in blue and the measured modulation amplitude in red for the continuous rotation (left) and the stepwise movement (right). Time is normalized to the period of the motion

\section{PRELIMINARY IN-CORE CALIBRATION}

The calibration aimed at establishing PISTIL's reactivity worth function vs. angle.

Two methods were tested and compared: modulation experiments during which PISTIL was operated at a constant speed and a step reactivity insertion experiments. During the experiments, the reactor was brought to criticality at a power of approximately $1 \mathrm{~W}$ while PISTIL remained static. The calibrations were then performed through either a reactivity modulation or a step reactivity variation.

\section{A. Experimental setup}

The experiments were conducted in two different manners. While the $\mathrm{B}_{4} \mathrm{C}$ control rods were fully extracted, the criticality was maintained by adjusting the core water level. Alternatively the water level remains constant when operating with the rods. The neutron instrumentation consisted in 4 Photonis CFUL01 fission chambers located symmetrically with respect to the center of the core as detailed in the reference configuration [9]. Their output currents were sampled at $1 \mathrm{kHz}$. Two additional Photonis CFUM21 detectors were also used for operation monitoring purposes.

The criticality water level reached in the experiments were compared to the operation histories of CROCUS without PISTIL installed. The overall reactivity effect introduced by PISTIL is approximately $-0.13 \$$. A precise estimation of the reactivity worth was be made due to differences in detector setups.

\section{B. Differential reactivity worth}

The response of the reactor with respect to reactivity modulation were measured during a constant frequency rotation at $1.110^{-2} \mathrm{~Hz}$. The resulting reactivity profile were estimated by inverse kinetic analysis [1] using values of adjoint-weighted kinetic parameters of CROCUS calculated in Tripoli4 [13] with JEFF 3.3 library. The reactivity as a function of the angular position of PISTIL obtained from the 4 signals are shown in Figure 8 . The solid lines represents the reactivity effects with a angular resolution of $1^{\circ}$ and the shaded area are the associated estimated uncertainties. The measured profiles were consistent between detectors. A maximum reactivity modulation amplitude of $1.35 \pm 0.26 \varnothing(10.24 \pm 1.97 \mathrm{pcm})$ was observed, while the angular position between the maxima and the minima 
was $90 \pm 1^{\circ}$. The results are consistent with the simulation predictions. However, a shift of approximately $30^{\circ}$ was observed between the expected position of the extrema (at 0 and $90^{\circ}$ ). This could be due to errors during the assembling process of the device that will be examined by out-of-core verifications.

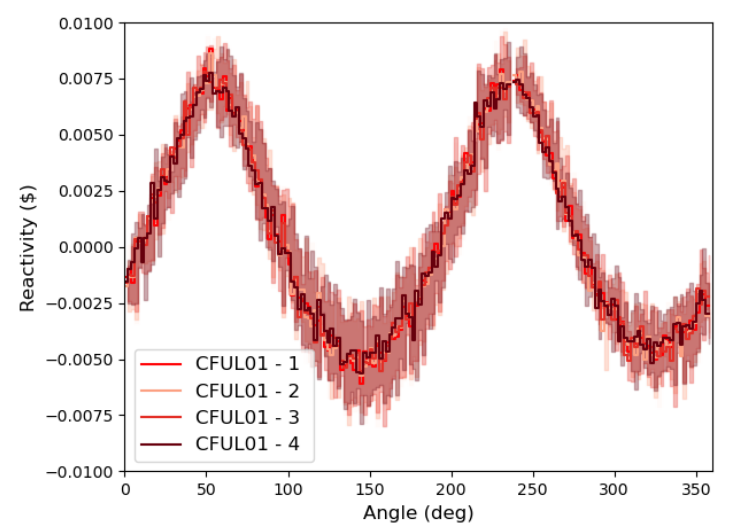

Figure 8. Measured reactivity profile of PISTIL with respect to its angular position from the dynamic calibration method

\section{Asymptotic period measurement}

Verification of the measured reactivity profile was also conducted using the asymptotic period method. The critical state water levels of PISTIL at $45^{\circ}$ and $135^{\circ}$ were first measured. Positive and negative reactor period were then measured during 30 minutes through a step reactivity variation from the critical state by the position switch of PISTIL (i.e. from $45^{\circ}$ to $135^{\circ}$ and vice versa)..The uncertainties are evaluated with respect to the determination of least-square fitted reactor periods. It was observed that for both positive and negative periods are of the same order of magnitude. The differences in the measurements could be related to the mechanical precision of the reactor operation and thus the difficulty in reaching the identical initial critical state. The resulting differential reactivities are approximately $8 \mathrm{pcm}$ when evaluated using the inhour equation. The values are consistent with the dynamic measurements.

TABLE III

ASYMPTOTIC PERIOD MEASUREMENT CONFIGURATIONS AND PRELIMINARY RESULTS

\begin{tabular}{ccccc}
\hline \hline $\begin{array}{c}\text { Core water level } \\
(\mathrm{mm})\end{array}$ & $\begin{array}{c}\mathrm{B}_{4} \mathrm{C} \text { rod } \\
\text { position } \\
(\mathrm{mm})\end{array}$ & $\begin{array}{c}\text { PISTIL } \\
\text { position }\left({ }^{\circ}\right)\end{array}$ & Period (s) & $\begin{array}{c}\text { Reactivity } \\
(\mathrm{pcm})\end{array}$ \\
\hline 984.0 & 1000.0 & 45 & $1044.0 \pm 0.8$ & 8.68 \\
981.9 & 135 & $-1096.1 \pm 0.9$ & -8.81 \\
\hline 990.0 & 715.0 & 45 & $1052.4 \pm 1.1$ & 8.58 \\
990.0 & 678.0 & 135 & $-1129.7 \pm 1.1$ & -8.54 \\
\hline \hline
\end{tabular}

\section{CONCLUSION AND OUTLOOKS}

The PISTIL device was developed for the in-core experimental study of reactor kinetics through reactivity modulation. Its design makes it possible to generate modulations in a large frequency range (from $1 \mathrm{mHz}$ to $200 \mathrm{~Hz}$ ). Its design was achieved by Monte Carlo calculations and adapted to a mechanical concept to be installed in the core center of the CROCUS reactor. The preliminary analysis of reactivity calibration tests in the CROCUS core showed a good consistency between Monte Carlo estimations and measurements. This suggests the validity of the developed design methodology with respect to the estimation of small reactivity variations through differential calculations. Further development will be performed towards better characterization of the device and the optimization of future experiments. The first experimental campaign for the measurement of the zeropower transfer function was carried out in June 2021 in CROCUS. Modulations covering the whole frequency range of the design were performed. A second campaign is under preparation in CROCUS and is expected in October 2021.

\section{ACKNOWLEDGMENT}

This work was partly funded by I3P (CEA, EdF, Framatome) and by the CNRS/Défi NEEDS project.

\section{REFERENCES}

[1] K.O. OTT, R.J. NEUHOLD, "INTRODUCTORY NUCLEAR REACTOR DYNAMICS”, AMERICAN NUCLEAR SOCIETY, 1985

[2] Y. YedVAB, I. Reiss, "DeterminAtion OF DElayed NEUTRONS SOURCE IN THE FREQUENCY DOMAIN BASED ON INPILE OSCILLATION MEASUREMENTS", IN PROCEEDING OF PHYSOR-2006, 2006, PP.1-9

[3] E. Gilad ET Al., "EXPerimental estimation of the DELAYED NEUTRON FRACTION $\beta_{\text {eff }}$ OF THE MAESTRO CORE IN THE MINERVE ZERO POWER REACTOR", J. NUCL. SCI. TECHNOL. 52, 2015, PP.1026-1033

[4] P. LECONTE ET AL., "NEW DELAYED NEUTRON GROUP CONSTANTS AND COVARIANCES FOR LWR CORE APPLICATIONS, COMBINING SUMMATION CALCULATIONS AND INTEGRAL EXPERIMENTS", ANN. NUCL. ENERGY, VOL.139, 2020

[5] D. Foligno, "New Evaluation of DELAYED-NEUtron DATA AND ASSOCIATED COVARIANCES", PH.D. DISSERTATION, AIX-MARSEILLE UNIVERSITY, 2019

[6] Y. JIANG ET AL., "REVIEW OF KINETIC MODULATION EXPERIMENTS IN LOW POWER NUCLEAR REACTORS", EPJ NUCLEAR SCI. TECHNOL. VOL. 6, 2020

[7] E. BRUn et AL., TRIPOLI-4®, "CEA, EDF AND AREVA REFERENCE MONTE CARLO CODE”, ANN. NUCL. ENERGY, VOL.82, PP. 151-160. 2015

[8] A. JEBb,. "KinetiC StUdies ON THE UNIVERSITY OF LONDON NUCLEAR REACTOR”, PH.D. DISSERTATION, IMPERIAL COLLEGE OF SCIENCE AND TECHNOLOGY, 1973

[9] U. KASEMEYER ET AL., "BENCHMARK ON KINETICS PARAMETERS IN CROCUS”, NEA/NSC/DOC. 2006

[10] Trio Motion Technology Ltd, "USING THE PC MOTION ACTIVEX CONTROL”, TRIO MOTION 
TeChNOLOGy Motion COORdinator - TeChNicAl REFERENCE MANUAL, 2008

[11] O. CABELLOS ET AL., "BENCHMARKING AND VALIDATION ACTIVITIES WITHIN JEFF PROJECT", EPJ WEB CONF. VOL.146, PP.4-9, 2017

[12] Elliott C et Al., "Instruments LabVieW: A PROGRAMMING ENVIRONMENT FOR LABORATORY AUTOMATION AND MEASUREMENT", JOURNAL OF THE ASSOCIATION FOR LABORATORY AUTOMATION, VOL.12. PP. 17-24, 2007

[13] Guillaume Truchet et Al.. "Computing adjointWEIGHTED KINETICS PARAMETERS IN TRIPOLI-4 BY THE ITERATED FISSION PROBABILITY METHOD", ANN. NUCL. ENERGY, VOL.85, 2015

[14] V. LAMIRAND ET AL., THE COLIBRI EXPERIMENTAL PROGRAM IN THE CROCUS REACTOR: CHARACTERIZATION OF THE FUEL RODS OSCILLATOR, EPJ WEB CONF. VOL.225, 2020 